\title{
LE RAPPORT MONDIAL DE L'UNESCO SUR LES SOCIÉTÉS DU SAVOIR. ÉTAT DES LIEUX AU QUÉBEC, DIX ANS PLUS TARD
}

\author{
JEAN BERNATCHEZ \\ Université du Québec à Rimouski (UQAR)
}

Résumé - Le rapport mondial de l'UNESCO sur les sociétés du savoir, publié en 2005, incarne l'idéal de la science au service du développement humain. Le modèle qui y est décrit constitue une solution de remplacement à celui de l'économie du savoir, proposé par l'OCDE en 1996, lequel inspire les politiques scientifiques des pays industrialisés. Nous reprenons dans cet article trois thèmes centraux du rapport de l'UNESCO sur les sociétés du savoir et nous déclinons les valeurs et les normes qui les orientent: la gouvernance de la science, l'organisation de la recherche scientifique et l'évolution de l'enseignement supérieur. Pour chaque thème, nous dressons un état des lieux au Québec selon l'idéal des sociétés du savoir.

$$
\div \div
$$

\section{The UNESCO World Report on Knowledge Societies.}

The Situation in Quebec, Ten Years Later

Abstract - Published in 2005, the UNESCO world report on knowledge societies embodies the ideal of science being at the service of human development. It provides an alternative to the model of the knowledge economy put forward by the OECD in 1996, which underpins the science policy of industrialized countries. This article explores three central themes of the UNESCO world report on knowledge societies and identifies the values and norms that guide them: the governance of science, the organization of scientific research, and the evolution of higher education. For each theme, I describe the current situation in Québec, in light of the ideal of knowledge societies.

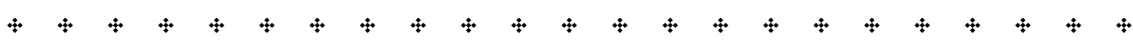


L'analyse des politiques scientifiques des pays industrialisés suggère que leur source d'inspiration est le modèle de l'économie du savoir ${ }^{1}$. Dans le vocabulaire de l'approche cognitive et normative d'analyse des politiques, ces modèles sont appelés référentiels ${ }^{2}$. L'approche postule que les politiques sont le fruit d'interactions sociales qui donnent lieu à la production d'idées et de valeurs communes. Les politiques sont liées à un paradigme sociétal ${ }^{3}$, à une vision $d u$ monde partagée, bref à un référentiel marqué par des valeurs et par des normes. Le référentiel est un espace de sens où se cristallisent les conflits entre les acteurs.

Le référentiel de l'économie du savoir se développe au milieu des années 1990. Le texte fondateur, intitulé L'économie fondée sur le savoir, est publié par l'Organisation de coopération et de développement économiques (OCDE) en $1996^{4}$. L'économie fondée sur le savoir repose sur la production, la diffusion et l'utilisation du savoir dans une perspective de croissance économique. L'importance du savoir comme capital humain est reconnue ${ }^{5}$, un capital qui s'acquiert par l'éducation, se préserve par la formation tout au long de la vie et rapporte des dividendes qui se mesurent par une augmentation de la productivité. Le savoir stimule la performance économique. L'OCDE propose une typologie des savoirs utile dans ce contexte: le savoirquoi renvoie à la connaissance factuelle; le savoir-pourquoi est lié à la connaissance des principes régissant les phénomènes; le savoir-faire fait référence aux compétences ; et le savoir-qui permet de repérer les experts et d'utiliser efficacement leurs connaissances.

Alors que le référentiel dominant postule l'existence d'une économie du savoir, un référentiel alternatif suppose le développement de sociétés du savoir, sociétés au pluriel, ce qui engage à la diversité culturelle. L'Organisation des Nations unies pour l'éducation, la science et la culture (UNESCO) en fait la promotion avec la publication, en 2005, du rapport intitulé Vers les sociétés du savoir ${ }^{6}$. Dans ces sociétés existe la capacité de

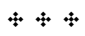

1. Cet article reprend certains extraits de notre thèse de doctorat, inédite: Jean BERNATCHEZ, "Référentiels et dynamiques des politiques publiques de l'organisation de la recherche au Québec ", thèse de doctorat (sciences de l'éducation), Université Laval, 2009.

2. Pierre MULLER, «L'analyse cognitive des politiques publiques. Vers une sociologie politique de l'action publique ", Revue française de science politique, vol. 50, n 2, 2000, p. 189-207.

3. Yves SUREL, "Les politiques publiques comme paradigmes", Alain FAURE, Gilles POLLET et Philippe WARIN, La construction du sens dans les politiques publiques, Paris, L'Harmattan, 1995, p. 125-151.

4. OCDE, L'économie fondée sur le savoir, Paris, OCDE, 1996.

5. Gary S. BECKER, Human Capital A Theoretical and Empirical Analysis with Special Reference to Education, New York, Columbia University Press, 1964.

6. UNESCO, Vers les sociétés du savoir, Paris, UNESCO, 2005. 
produire, de diffuser et d'utiliser l'information en vue d'appliquer les savoirs au développement humain. La liberté d'expression et la coopération en sont les fondements. L'accès universel à la connaissance est un préalable, ce qui suppose des stratégies de lutte contre la pauvreté. La commercialisation des résultats de la recherche restreint l'accès à la connaissance; aussi, il importe d'adopter une voie intermédiaire conciliant le droit au savoir et la protection de la propriété intellectuelle. Il faut considérer de manière équilibrée ces différents savoirs : descriptif (les faits), procédural (le comment), explicatif (le pourquoi) et comportemental (le vivre-ensemble).

Le rapport de l'UNESCO propose un idéal qui compose avec de nombreuses contraintes, entre autres la prégnance du référentiel de l'économie du savoir présenté comme pragmatique et adapté aux caractéristiques du marché mondialisé dans lequel les États, réduits à des unités économiques, rivalisent pour obtenir plus de ressources. Le vocabulaire entrepreneurial caractérise le discours : "cette technique rhétorique qui discrédite une valeur référentielle pour mieux la dépasser permet $[. .$.$] de conférer aux projets de$ réforme en vigueur une dimension d'amélioration [...] mais aussi de leur attribuer un statut de nécessité ${ }^{\prime}$. Aline Giroux l'associe à une novlangue: "La procédure d'enfermement de la pensée commence par la création d'un discours qui devient dominant parce qu'il est uniformément repris [...], qu'il présente les choses à partir d'un seul point de vue ${ }^{8}$ ». La performance est présentée comme une vertu: seuls ceux qui ne s'imposent pas cette rigueur la critiquent. Ce discours exclut tout contre-argument : les scientifiques sont avant tout des experts qui doivent répondre aux besoins nationaux.

Les référentiels sont des idéaux types au sens où l'entend Max Weber: des productions idéalisées utiles à la compréhension. On ne les retrouve pas intégralement dans l'univers social, mais ils permettent de rendre compréhensibles les phénomènes. De plus, les discours de l'OCDE et de I'UNESCO tendent récemment à être plus nuancés, à s'inspirer mutuellement. Les deux organisations partagent plusieurs forums internationaux. L'OCDE est maintenant moins cantonnée dans un économisme exclusif et l'UNESCO devient plus pragmatique.

Nous retenons ici trois thèmes centraux du rapport de l'UNESCO sur les sociétés du savoir et nous déclinons les valeurs (principes qui guident

$$
\div+\div
$$

7. Kenneth Bertrams, "Les interactions industrie-université. Essai de repérage historique», Yves GINGRAS et Lyse RoY (dir.), Les transformations des universités du XIIT au XXI siècle, Québec, Presses de l'Université du Québec, 2006, p. 90.

8. Aline GirouX, Le pacte faustien de l'université, Montréal, Liber, 2006, p. 147. 
l'action) et les normes (exemples de ce qui est conforme à un idéal) qui les orientent: la gouvernance de la science, l'organisation de la recherche scientifique et l'évolution de l'enseignement supérieur. Pour chaque thème, nous dressons aussi un état des lieux au Québec considérant l'idéal des sociétés du savoir. L'approche cognitive et normative d'analyse des politiques est notre cadre de référence théorique. Sur le plan méthodologique, les techniques d'analyse documentaire, d'entrevues avec les détenteurs d'enjeux et d'observation participante sont mises à contribution.

\section{LA GOUVERNANCE DE LA SCIENCE}

Le rapport de l'UNESCO invite à revoir les relations entre la science et la société. Cela relève de la gouvernance. Ce concept entretient avec celui de gouvernement un rapport d'opposition : la notion de gouvernance «émerge face au diagnostic d'une incapacité des gouvernements à répondre aux problèmes $[. .$.$] et à s'ajuster à de nouvelles formes d'organisation " ». Le concept$ de gouvernement décrit une autorité contraignante exercée par l'État, alors que celui de gouvernance propose une forme souple de pouvoir caractérisée par les interactions entre les acteurs.

Le concept de gouvernance n'est pas lié à une école de pensée, mais les économistes néoclassiques l'associent à l'efficience (efficacité au moindre coût). Cela induit que les modalités d'action publique sont privilégiées au détriment des finalités. Il est possible, toutefois, de définir la gouvernance comme un "processus de coordination d'acteurs [...] en vue d'atteindre des objectifs définis et discutés collectivement ${ }^{10}$ ". Ce processus se caractérise par le polycentrisme institutionnel (plusieurs lieux de décision et d'exécution), une frontière poreuse entre le privé et le public et un rapport à l'autorité peu contraignant ${ }^{11}$. Pour l'UNESCO, la gouvernance de la science se fait au moyen de la «définition de normes, [ce] qui est notamment le fait des instances gouvernementales [...] mais [...] doit en outre s'accompagner d'une sensibilisation du public ${ }^{12}$ ».

L'UNESCO milite pour une nouvelle forme de gouvernance de la science, le modèle du public avec décisions partagées plutôt que le modèle classique avec décisions linéaires. Dans le modèle classique, l'État et l'entre-

$$
4+4
$$

9. Patrick Le GALĖs, « Gouvernance », Laurie BOUSSAGUET et al. (dir.), Dictionnaire des politiques publiques, Paris, Presses de Sciences Po, 2010, p. 300.

10. Ibid., p. 301.

11. Ibidem.

12. UNESCO, Vers les sociétés du savoir..., op. cit., p. 123. 
prise sont en relation quasi exclusive avec les lieux de la science (la société civile est un acteur passif). Avec le modèle du public, l'image du triangle est évoquée (lieux de la science, État et entreprises, société civile) avec, à l'interface, un espace public de débat, de concertation ou de confrontation. Qu'en est-il de la situation au Québec pour les initiatives gouvernementales et celles de la société civile? La science est de juridiction partagée entre les gouvernements provincial et fédéral. C'est avec leurs politiques scientifiques que ceuxci définissent les valeurs et les normes de gouvernement ou de gouvernance de la science.

Au Québec, Savoir changer le monde, la politique scientifique rendue publique par le gouvernement du Parti québécois en 2001, oriente d'abord l'action ${ }^{13}$. Elle entend favoriser le mieux-être de la société québécoise, concourir à la prospérité dans une perspective de développement durable, enrichir la culture québécoise et contribuer au patrimoine mondial des connaissances. Elle est organisée selon trois axes : la formation des personnes et le partage démocratique du savoir, le développement des connaissances et l'innovation technologique et sociale. Les modalités sont orientées vers la valorisation commerciale de la recherche, mais la préoccupation pour l'innovation sociale (dont la finalité est le bien commun) et la volonté de partage démocratique du savoir fondent un cadre susceptible de prendre en compte le modèle de gouvernance promu par l'UNESCO. Toutefois, le concept d'innovation sociale est difficilement opératoire et la notion de partage démocratique des savoirs demeure de l'ordre de la rhétorique ${ }^{14}$.

Le gouvernement du Parti libéral assume le pouvoir à compter de 2003 et propose une révision de cette politique en publiant Un Québec innovant et prospère (2006) ${ }^{15}$ et Mobiliser, innover, prospérer (2010) ${ }^{16}$. Ces deux stratégies définissent une orientation marquée vers la valorisation commerciale de la recherche et mettent en jachère la volonté d'innovation sociale et la dimension du partage du savoir. À compter de 2012, le gouvernement entend proposer une nouvelle stratégie en misant cette fois sur la consultation de quelques acteurs clés.

$$
4+4
$$

13. Ministère DE la ReCherChe, de la SCIENCE ET DE LA TeChnOlogie, Savoir changer le monde, Québec, MRST, 2001.

14. Jean BERNATCHEZ, "Social Innovation and the University in Quebec. The Challenge to Operationalize a Polysemic Concept", Hebe Vessuri et Ulrich TeICHLER (dir.), Universities as Centres for Research. An Endangered Species?, Rotterdam, Sense, 2008, p. 179-187.

15. Ministère DU DÉVELOPPEMENT ÉCONOMIQUE, DE L'INNOVATION ET DE L'EXPORTATION, $U n$ Québec innovant et prospère, Québec, MDEIE, 2006.

16. Ministère DU DÉVELOPPEMENT ÉCONOMIQUE, DE L'INNOVATION ET DE L'EXPORTATION, Mobiliser, innover, prospérer, Québec, MDEIE, 2010. 
À ce moment, le Parti libéral perd le pouvoir aux mains du Parti québécois, qui élargit la consultation et propose une nouvelle politique scientifique intitulée Priorité Emploi (2013) ${ }^{17}$ à l'enseigne suivante : la recherche du bien commun, la quête de l'excellence et le partage d'une culture scientifique. Deux principes guident l'action : l'équilibre entre tous les types de recherche, fondamentale ou appliquée, libre ou orientée; l'importance de la collaboration et de la concertation de tous les acteurs. La politique dit placer l'humain au centre de ses préoccupations. Dans le contexte de l'élaboration de cette politique et dans celui des cinq chantiers consécutifs au Sommet sur l'enseignement supérieur, le gouvernement mise sur des méthodes consultatives et délibératives adaptées au modèle du public avec décisions partagées.

Cette politique n'est pas déployée lorsque le Parti libéral reprend le pouvoir en 2014. Il est possible que ce gouvernement reconduise sans enthousiasme la politique de 2013 (c'est ce qui se produit lors d'un changement de gouvernement pour les politiques complexes), mais il figure, au nombre de ses engagements, des modalités semblables à celles des stratégies de 2006 et 2010 qui visent plutôt une augmentation des revenus des universités par la commercialisation des brevets, misant sur la privatisation et délaissant l'option du partage du savoir. Au moment d'écrire ces lignes, c'est toujours le "silence radio" en ce qui concerne les intentions gouvernementales ${ }^{18}$.

Au Canada, la politique scientifique de 1996 du gouvernement libéral, Les sciences et la technologie à l'aube du XXI siècle, "repose sur le postulat de l'évolution des pays industrialisés du monde entier vers une économie du savoir ${ }^{19}$ ". Le plan veille à l'application et à la commercialisation des sciences et des technologies en vue de la croissance économique. Dans ce contexte sont néanmoins créés des instruments à portée plus universelle : la Fondation canadienne pour l'innovation (1997), les Bourses du millénaire (1998), les Chaires de recherche du Canada (2000) et le financement des frais indirects de recherche (2001).

En 2002, ce même gouvernement publie une nouvelle politique scientifique, Atteindre l'excellence ${ }^{20}$, qui propose quatre défis : la performance

$$
+4
$$

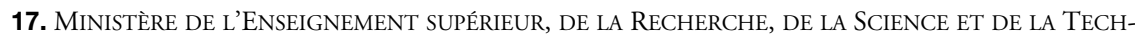
NOLOGIE, Priorité Emploi, Québec, MESRST, 2013.

18. Étienne PLAMONDON ÉMOND, "Une recherche de moins en moins distincte», Le Devoir, 21 février 2015, http://www.ledevoir.com/societe/science-et-technologie/432191/une-recherche-de-moins-enmoins-distincte (7 mars 2015).

19. GOUVERNEMENT DU CANADA, Les sciences et la technologie à l'aube du XXI siècle, Ottawa, Gouvernement du Canada, 1996, p. 1.

20. Industrie Canada, Atteindre l'excellence, Ottawa, Industrie Canada, 2002. 
(trouver de meilleures façons de créer les connaissances et de commercialiser les idées), les compétences (trouver de meilleurs moyens de former et d'attirer les plus brillants chercheurs), l'innovation (trouver des moyens d'améliorer les politiques afin de favoriser l'innovation) et les collectivités (encourager l'innovation à l'échelle locale). Le gouvernement s'engage à doubler le budget des conseils subventionnaires à l'horizon 2010, mais on subodore une volonté d'orienter la recherche vers des finalités économiques, puisqu'une des cibles de la politique est de tripler en huit ans les résultats de la valorisation commerciale de la recherche.

En 2007, le nouveau gouvernement conservateur propose une stratégie intitulée Réaliser le potentiel des sciences et de la technologie au profit $d u$ Canada $^{21}$, qui s'inscrit comme suite au plan économique Avantage Canada - Bâtir une économie forte pour les Canadiens ${ }^{22}$. Cinq avantages sont préalables à l'action : l'avantage fiscal (taux d'imposition le plus bas des pays du G-7 sur les nouveaux investissements des entreprises), l'avantage financier (élimination de la dette publique), l'avantage entrepreneurial (réduction de la réglementation), l'avantage du savoir (formation de la main-d'œuvre la plus souple du monde) et l'avantage infrastructurel (création d'une infrastructure assurant la libre circulation). Le gouvernement veut concentrer ses efforts dans les domaines d'intérêt national, conscient du rôle que jouent la science et la technologie dans la consolidation d'une économie concurrentielle.

Ce discours est le cadre de légitimation des actions du gouvernement canadien en sciences, mais les conservateurs improvisent sur ce thème imposé, ce qui n'est pas conforme à la tradition : «Les Canadiens, qui ont l'habitude de voir leurs gouvernements, même conservateurs, gouverner pas trop loin du centre, semblaient croire qu'il en serait de même sous Harper. Grave erreur ${ }^{23}$ !» Chris Turner dresse le bilan des dérives à ce chapitre, qu'il interprète comme une guerre livrée à la science et à l'esprit des Lumières : «La seule vérité [...], ce n'est pas celle qui découle d'une recherche scientifique indépendante et de haut niveau, mais celle que lui dicte son idéologie de droite crypto-évangéliste ${ }^{24}{ }^{2}$. Depuis 2007 , les actions

$$
\div \div
$$

21. INDUSTRIE CANADA, Réaliser le potentiel des sciences et de la technologie au profit du Canada, Ottawa, Industrie Canada, 2007.

22. MINISTĖRE DES FINANCES, Avantage Canada. Bâtir une économie forte pour les Canadiens, Ottawa, Ministère des Finances, 2006.

23. Yves GIngras, "Le réveil des scientifiques", Chris TURnER, Science on coupe!, Montréal, Boréal, p. 12-13.

24. Chris TURNER, Science on coupe!, Montréal, Boréal, jaquette. 
canadiennes en science surprennent : protocole de communication en vertu duquel les scientifiques des ministères doivent transmettre les demandes des médias à des conseillers en communication et fournir des réponses préapprouvées (2007); nomination d'un ministre d'État à la Science et à la Technologie d'idéologie créationniste (2008); rejet des ententes sur la mise en œuvre du protocole de Kyoto (2009) ; abolition du questionnaire détaillé du recensement, ce qui rend moins fiables les données de Statistique Canada (2010) ; projet de loi omnibus C-38, qui révise des lois clés et prévoit la fermeture de plusieurs installations de recherche de premier plan (2012).

En 2014, Ottawa lance une consultation sur la prochaine stratégie scientifique ${ }^{25}$, mais en orientant les questions autour des recommandations d'un groupe d'experts. Celui-ci réunit Jenkins, président de Open Text Corporation, une société de logiciels ; Gupta, président-directeur général de MITACS, un réseau qui regroupe les secteurs privé et public «dans le but d'élaborer des outils pour l'économie du savoir "; Dahlb, professeur d'économie associé à l'Institut Fraser, un think tank de la droite libertarienne; Leroux, présidente du Mouvement Desjardins; Naylor, recteur de l'Université de Toronto ; et Robinson, présidente de Polytechnics Canada, un regroupement d'instituts technologiques ${ }^{26}$.

Leurs recommandations visent à améliorer l'accès au capital de risque, à affecter les ressources gouvernementales à un petit nombre de programmes directs d'innovation dans les entreprises et à restructurer le Conseil national de recherches du Canada. Avec moins d'un mois pour le faire, les Canadiens sont conviés à répondre à cinq questions, dont celles-ci :

Selon les conseils fournis par le groupe d'experts, que peut-on faire de plus pour améliorer les investissements des entreprises en recherche et développement? Quelles mesures peuvent être prises pour améliorer la mobilisation des connaissances des établissements d'enseignement supérieur vers le secteur privé?

Les finalités sont campées, les Canadiens sont consultés sur les seules modalités dans un cadre qui ne favorise pas le dialogue. Borde résume:

Ottawa ne veut vous entendre ni sur les domaines de recherche qui seraient à privilégier ni sur les besoins de la société (autre que celui des retombées économiques) [...] Pas plus sur comment on forme des

$$
4+4
$$

25. INDUSTRIE CANADA, Un moment à saisir pour le Canada, Ottawa, Industrie Canada, 2014.

26. INDUSTRIE CANADA, "Consultation sur la science et la technologie», http://www.ic.gc.ca/eic/site/ icgc.nsf/fra/h_07416.html (20 avril 2014). 
esprits éclairés grâce à la science. On veut juste savoir ce que vous pourriez faire de plus pour que l'industrie canadienne innove et en tire des bénéfices ${ }^{27}$.

La stratégie rendue publique en 2014, Aller de l'avant dans le domaine des sciences, de la technologie et de l'innovation, "place l'innovation au premier plan - en favorisant l'innovation dans les entreprises, en créant des synergies avec les capacités de recherche du Canada, et en utilisant la main-d'œuvre qualifiée et novatrice de notre pays ${ }^{28}$ ".

Une politique, ce sont des idées inscrites dans un document normatif, mais ce sont des "idées en action ", pour reprendre l'expression consacrée par l'approche cognitive et normative d'analyse des politiques. L'action du gouvernement conservateur est à mille lieues du modèle de gouvernance promu par l'UNESCO. Elle s'inscrit dans un modèle de gouvernement qui exerce son autorité de manière hiérarchique, contraignante et dogmatique, alors que, dans la société civile, on remarque pourtant un tournant délibératif en ce qui concerne les rapports entre science et société.

Florence Piron ${ }^{29}$ l'observe à partir des années 1980 dans un contexte d'alertes et de crises sanitaires, entre autres Bophal (1984), Tchernobyl (1986) et le scandale du sang contaminé (1986), mais ce tournant délibératif est également inspiré par quelques ouvrages phares : Le principe responsabilité de Hans Jonas (1979), La société du risque d'Ulrich Beck (1986) et le rapport Brundtland de la Commission mondiale sur l'environnement et le développement (1987) ${ }^{30}$. Au Québec, les initiatives consultatives et délibératives sont diversifiées, mais en marge des politiques scientifiques : des états généraux et des consultations en commissions parlementaires, des audiences publiques, le projet Perspectives STS du Conseil de la science et de la technologie (organisme consultatif aboli en 2011) et les initiatives de l'Institut du Nouveau Monde (créé en 2004) ${ }^{31}$. À cela s'ajoutent celles de

$$
4 \div
$$

27. Valérie BORDE, "Stratégie scientifique fédérale, une consultation?", Blogue de L'Actualité, 20 janvier 2014, http://www.lactualite.com/blogues/le-blogue-sante-et-science/politique-scientifiquefederale-ca-une-consultation/ (20 janvier 2014).

28. INDUSTRIE CANADA, Aller de l'avant dans le domaine des sciences, de la technologie et de l'innovation, http://www.ic.gc.ca/eic/site/icgc.nsf/fra/h_07472.html (7 mars 2015).

29. Florence PIRON, "Les transformations récentes des rapports science et société dans le monde", Léonore PION et Florence PIRON (dir.), Aux sciences, citoyens!, Montréal, Presses de l'Université de Montréal, 2009, p. 11-17.

30. Hans JONAS, Le principe responsabilité. Une éthique pour la civilisation technologique, Paris, Cerf, 1979; Ulrich BECK, Risikogesellschaft. Auf dem Weg in eine andere Moderne, Berlin, Suhrkamp, 1986 (en français : La Société du risque. Sur la voie d'une autre modernité, Paris, Aubier, 2001) et la COMMISSION DES NATIONS UNIES SUR L'ENVIRONNEMENT ET LE DÉVELOPPEMENT, Notre avenir à tous (Rapport Brundtland), Paris, Commission des Nations Unies sur l'Environnement et le Développement, 1987.

31. Léonore PION et Florence PIRON, Aux sciences, citoyens!..., op. cit., p. 65-141. 
l'Association science et bien commun (créée en 2011), qui a pour mission de stimuler la vigilance et l'action pour une science publique au service du bien commun.

\section{L'ORGANISATION DE LA RECHERCHE SCIENTIFIQUE}

Les concepts de la systémique (activités, acteurs, environnements, finalités, évolution) sont utiles à la définition de ce qu'est la recherche scientifique : une dynamique collective constituée d'activités structurées et normées (normes scientifiques et éthiques) visant la production de nouveaux savoirs (savoir-faire et savoir-être), conduites par des acteurs (les scientifiques, mais aussi d'autres personnels), s'inscrivant dans le cadre de domaines du savoir (ou de disciplines) et dont les résultats sont validés et rendus publics, le tout évoluant dans le temps.

La recherche est «un processus finalisé par la publication et la mise à l'épreuve publique des résultats ${ }^{32}$ ». Cette définition procédurale montre la nécessité qu'une recherche particulière conduise à une production qui devra être évaluée. Le terme processus suppose l'idée d'une série de phénomènes qui se succèdent de la même manière. Ce n'est pas le cas de la recherche scientifique considérée de façon générale, surtout depuis l'abandon de la logique de linéarité caractéristique des premières versions du Manuel de Frascati de l'OCDE, référence méthodologique de la mesure des activités de recherche et développement. La dynamique envisage plutôt les phénomènes dans leur mouvement. Ainsi, il n'est pas possible de parler du caractère finalisé de la recherche, il faut plutôt inscrire le phénomène dans le cadre d'une quête permanente orientée vers l'actualisation de certaines finalités.

Les caractéristiques du référentiel des sociétés du savoir promu par l'UNESCO sont en adéquation avec la mission de cette "organisation intellectuelle» des Nations unies: "À l'heure où le monde cherche des voies nouvelles pour construire la paix et le développement durable, nous devons compter sur le pouvoir de l'intelligence à innover, à élargir nos horizons pour faire vivre l'espoir d'un nouvel humanisme ${ }^{33}$ ». L'UNESCO est un laboratoire d'idées, un organisme normatif, mais aussi un catalyseur de coopération internationale orienté vers la paix et le développement. Son message rend

$$
4+4
$$

32. Camille LIMOGES, "L'université à la croisée des chemins. Une mission à affirmer, une gestion à réformer ", Le lien formation-recherche à l'université, Montréal, Conseil de la science et de la technologie et ACFAS, 1996, p. 20.

33. UNESCO, "Ä propos de l'UNESCO», https://fr.unesco.org/about-us/propos-de-lunesco (20 avril 2014). 
compte d'une finalité, actualisée au rythme des mouvements qui secouent le monde, s'exprimant par ces deux dimensions : paix et développement.

Les sociétés sont mondialisées et métissées; aussi le dialogue interculturel est-il une nécessité pour développer le vivre-ensemble. Le monde est incertain, l'avenir dépend de la capacité collective à mieux comprendre les phénomènes naturels et culturels : cela passe par l'éducation, la recherche scientifique et le partage des savoirs. Le monde est instable, les sociétés sont fragilisées par des risques multiples. Le respect des droits fondamentaux est garant de la stabilité des sociétés : «Face à l'émergence [...] des sociétés du savoir [...] la participation active de chacun au nouvel espace public mondial est une condition de la paix et du développement $t^{34}$ ».

La recherche scientifique est un des lieux où s'édifient les sociétés du savoir. Or il existe une fracture scientifique qui sépare les pays riches en sciences des autres: "L'idée qu'il y aurait deux mondes de la science [...] est un anathème contre l'esprit scientifique ${ }^{35}$ ». Partage et collaboration sont les vecteurs permettant de fonder les sociétés du savoir. De manière opératoire, sur le plan du partage, l'UNESCO insiste sur la mise à la disposition des chercheurs du monde entier des publications scientifiques : «L'Internet et la publication électronique rendent possible la création de bibliothèques scientifiques publiques contenant l'intégralité des textes et les données de n'importe quel article publié, accessible à chacun de partout et libre de droits ${ }^{36}$ ». Sur le plan de la collaboration, l'idée avancée par l'UNESCO est celle du collaboratoire: «Formé à partir des termes collaboration et laboratoire, le terme désigne l'ensemble des techniques, outils et équipements permettant à des scientifiques [...] de travailler avec des installations et des collègues situés à des distances qui auparavant rendaient difficiles les entreprises collectives ${ }^{37}$ ».

Paix et développement sur le plan des finalités de la recherche scientifique, partage et collaboration sur le plan des modalités, telle est "l'organisation ${ }^{38}$ " de la recherche scientifique proposée dans Vers les sociétés $d u$ savoir. C'est une utopie, certes, mais la communauté des chercheurs s'est

$$
+4
$$

34. Ibidem.

35. UNESCO, Vers les sociétés du savoir..., op. cit., p. 101.

36. Ibid., p. 119.

37. Ibid., p. 111.

38. L'organisation est le visage intériorisé du système, le système est le visage extériorisé de l'organisation (Edgar Morin, La Méthode. La connaissance de la connaissance, Paris, Seuil, 1977, p. 103 et 145). 
aussi constituée sur la base d'une utopie qui s'incarne à travers les normes du sociologue Merton ${ }^{39}$, lesquelles constituent l'ethos de la science ${ }^{40}$.

Ces normes sont l'idéal-type d'une communauté scientifique : "toute violation majeure de ces normes ne peut se faire qu'au détriment d'une bonne science ${ }^{41}$ ". La première norme est l'universalisme: les énoncés soumis doivent être évalués selon des critères impersonnels, sans égard aux caractéristiques du scientifique qui les propose. La deuxième norme est le communalisme: une découverte scientifique est un bien commun que ne peut s'approprier quelque individu ou collectivité. Le désintéressement constitue la troisième norme: le scientifique cherche la vérité et non le profit ou la gloire. Le scepticisme organisé, la quatrième norme, «enjoint le savant à avoir une attitude critique face à tout énoncé nouveau qui doit alors être scruté, vérifié et reproduit avant d'être accepté comme valide et intégré au savoir ${ }^{42}$ ». S'ajoute une norme d'humilité.

Cet idéal s'incarne dans le modèle de la République de la science, le référentiel dominant qui précède celui de l'économie du savoir. Avant 1980, les scientifiques disposent en effet d'un haut degré d'autonomie ${ }^{43}$. Polanyi définit ainsi cette conception : "the pursuit of science by independent self-co-ordinated initiatives assures the most efficient possible organization of scientific progress [...] any authority which would undertake to direct the work of the scientist centrally would bring the progress of science virtually to a standstill ${ }^{44}$ ". Une main invisible guide la coordination des activités de chaque scientifique par ajustements mutuels. Le mérite est lié à trois critères : la plausibilité, la valeur scientifique et l'originalité. L'évaluation par des pairs, sur la base de la qualité, conditionne la publication et l'octroi de ressources. Pour qu'il soit citoyen de cette république, les compétences d'un chercheur doivent être reconnues par ses pairs.

La recherche empirique en sociologie des sciences démontre cependant que les normes mertoniennes sont transgressées, bien qu'elles constituent l'idéal à atteindre. C'est vrai dans le contexte du référentiel de la

$$
4+4
$$

39. Jean-Claude GUÉDON, «Le libre accès et la grande conversation scientifique», Michaël E. SINATRA et Marcello Vitali-Rosai (dir.), Pratiques de l'édition numérique, Montréal, Presses de l'Université de Montréal, 2014, p. 111-126.

40. Jean BERNATCHEZ, "Le libre accès aux articles scientifiques. Référentiels, principes, normes et modalités ", Documentation et bibliothèques, vol. 61, $\mathrm{n}^{\circ} 1,2015$, p. 6-14.

41. Yves GINGRAS, Sociologie des sciences, Paris, Presses universitaires de France, 2013, p. 58.

42. Ibid., p. 55.

43. James Mullin, «Évolution des modes de financement de la recherche", Revue internationale des sciences sociales, $\mathrm{n}^{\circ} 168,2001$, p. 269-295.

44. Michael POLANYI, "The Republic of Science. Its Political and Economic Theory", Minerva, vol. 1, $\mathrm{n}^{\circ} 1,1962$, p. 56. 
République de la science comme dans celui de l'économie du savoir. Cependant, la nouvelle donne de l'économie du savoir fait que certaines normes, notamment le communalisme et le désintéressement, sont non seulement transgressées mais niées.

La mondialisation, définie comme la dynamique qui contribue à accroître les échanges entre les individus, les entreprises et les sociétés du monde, n'est pas une réalité nouvelle. Le phénomène de mondialisation est cependant récent. Levitt propose ce terme en 1983 (globalization) pour illustrer la convergence des marchés partout dans le monde ${ }^{45}$. Dans le champ scientifique, l'évènement marquant est l'adoption du Bayh-Dole Act par le Congrès des États-Unis en 1980, une loi permettant aux universités de breveter leurs découvertes réalisées grâce au financement public afin de favoriser le transfert technologique. "Cela va lancer les universités - tout d'abord aux États-Unis puis dans le monde entier - dans une course à la propriété intellectuelle qui va se révéler contraire aux logiques de partage des savoirs [...] qui avaient très largement cours auparavant dans la recherche ${ }^{46} »$.

Il est désormais question d'appropriation privée du savoir puisqu'il a une valeur stratégique dans un contexte de compétitivité accrue entre les espaces nationaux et internationaux. Les normes de communalisme et de désintéressement sont rejetées du nouvel univers éthique en construction : une éthique des moyens balisée par les principes et les normes des organismes subventionnaires et des comités d'éthique de la recherche (comment faire les choses) a préséance sur une éthique des fins (pourquoi faire les choses) associée à une morale qui n'a pas sa place en sciences. Comment ces constats se traduisent-ils au Québec?

D'emblée, rappelons que les référentiels sont des idéaux-types qui ne se retrouvent pas tels quels dans l'univers social. La réalité est plus complexe, plus subtile. Les organisations sont paradoxales, au sens où elles conjuguent parfois des finalités incompatibles. Le témoignage d'un professeur de sociologie québécois permet de rendre compte du phénomène ici et maintenant ${ }^{47}$.

Jean-Marc Fontan traite de la transformation de l'organisation de la recherche scientifique au Québec en s'inspirant d'auteurs classiques

$$
4 \div 4
$$

45. Theodore LEVITT, "The Globalization of Markets », Harvard Business Review, mai-juin 1983, p. 92 102.

46. Mélanie Dulong de Rosnay et Hervé Le Crosnier, Propriété intellectuelle. Géopolitique et mondialisation, Paris, Centre national de recherche scientifique, 2013, p. 148.

47. Jean-Marc FONTAN, "De l'intellectuel critique au professionnel de service», Cahiers de recherche sociologique, $\mathrm{n}^{\circ} 34,2000$, p. 79-97. 
(Joseph Schumpeter, John Dewey), contemporains (Antony Giddens, Alain Touraine) et de son expérience de chercheur explorant la voie de l'innovation sociale. Il croit que la recherche adopte les valeurs d'un projet modernisateur obéissant à une rationalité économique. Cela s'observe dans la taylorisation des activités de recherche et la quête d'efficience : "Concurrence oblige, l'université s'est transformée en une organisation économique soumise à l'exigence de rentabilité et ayant l'obligation d'afficher une bonne productivitét ${ }^{4}$. Dès le XIX ${ }^{e}$ siècle, John Dewey anticipe les dangers qui guettent une institution associée de trop près aux intérêts des pouvoirs politiques ou économiques. L'autonomie institutionnelle et la liberté universitaire sont garantes de la fonction civilisatrice de l'institution. Jean-Marc Fontan observe que les chercheurs travaillent sur des segments de la chaîne de connaissances. Ils deviennent des experts offrant des produits et des services. L'auteur tempère cependant le déterminisme de cette thèse par la reconnaissance d'îlots de résistance. Il présente son expérience de chercheur privilégiant une conception pragmatique du changement, qui fonde sa volonté de travailler en mode de recherche-action, soit « un processus de recherche construit avec des acteurs sur le terrain autour de préoccupations ou d'interrogations rejoignant des objectifs pratiques et universitaires ${ }^{49}$ ".

Les valeurs et les normes des politiques scientifiques du Québec et du Canada traduisent une volonté d'instrumentalisation de la recherche aux fins de servir les intérêts nationaux, souvent définis en termes économiques. On observe un glissement de la fonction gouvernementale de régulation de la recherche scientifique depuis la mission éducative vers la mission économique, pour preuve, les ministères de tutelle de la science: Industrie Canada et un ministère à vocation économique au Québec, généralement au moment où le Parti libéral forme le gouvernement, quoique le ministère de l'Éducation intègre depuis février 2015 les dimensions «enseignement supérieur et recherche ». Toutefois, les exemples de partage et de collaboration comme vecteurs d'une recherche scientifique orientée vers la paix et le développement sont légion. La recherche scientifique est de plus en plus orientée, mais elle l'est parfois dans un sens qui sert le bien commun, un concept que Jean-Paul Jouary définit ainsi : "le fait d'agir en commun pour dépasser ensemble ce qui fait obstacle au progrès de tous et à l'épanouissement de chacun $^{50}$ ".

$$
4+4
$$

48. Ibid., p. 82.

49. Ibid., p. 91.

50. Jean-Paul Jouary, cité dans Françoise DAVID, Bien commun recherché, Montréal, Écosociété, 2004, p. 33. 
De manière institutionnalisée, on observe la généralisation, au Québec, des dépôts d'universités qui rendent disponibles universellement les publications de leurs professeurs ${ }^{51}$ : le Service aux collectivités de l'Université du Québec à Montréal, qui, depuis 1979, permet à des groupes communautaires de profiter de services de recherche ou de formation; Accès savoir, la boutique des sciences de l'Université Laval; ou encore les groupes de recherche d'intérêt public de certaines universités, ouverts aux étudiants qui veulent agir sur des enjeux sociaux ou environnementaux. Force est toutefois d'admettre que les "projets témoignent davantage de l'engagement individuel de certains chercheurs en faveur d'une science publique que d'un choix collectif des universités, qui semblent plutôt avoir adopté la vision entrepreneuriale de la science prônée par les gouvernements ${ }^{52}$ ». Une étude que nous menon ${ }^{53}$ tend d'ailleurs à démontrer que la jeune génération de professeurs est plus encline que la génération précédente à adhérer aux principes et aux normes de l'économie du savoir. Formés dans ce cadre, les nouveaux professeurs intègrent mieux les normes de concurrence et de privatisation des savoirs. Quant à l'idée de collaboratoire, le terme n'est pas inscrit dans le langage courant, mais sa réalité est manifeste, à cette différence qu’y participent surtout les chercheurs " excellents" des "grandes universités de recherche", ainsi qualifiées à la suite d'une hiérarchisation des lieux de recherche, une tendance lourde depuis dix ans, excluant du coup plusieurs institutions et chercheurs de la périphérie. L'ouvrage de Lacroix et Maheu sur les grandes universités de recherche est éloquent à propos de ce mouvement promu par ces auteurs québécois, qui se définissent comme professeurs et recteurs émérites, «anciens hauts dirigeants d'une grande université de recherche ${ }^{54}$ ».

\section{L'ÉVOLUTION DE L'ENSEIGNEMENT SUPÉRIEUR}

Le rapport de l'UNESCO sur les sociétés du savoir fait état que, dans la plupart des pays, l'enseignement supérieur est constitué d'un réseau d'institutions publiques et privées. Le financement public garantit une certaine égalité des chances, mais sa remise en question ouvre la voie à des formes

$$
\div \div
$$

51. Jean BeRnATCHEZ, "Les dépôts institutionnels des universités québécoises. État des lieux ", Argus, vol. $43, n^{\circ} 1$, p. 24-27.

52. Florence PIRON, «De la culture scientifique à la démocratie scientifique», Léonore PION et Florence PIRON, Aux sciences, citoyens!..., op. cit., p. 69.

53. Frédéric Deschenaux et Jean Bernatchez, étude en cours sur le volet «recherche" de la tâche des professeurs.

54. Robert LACROIX et Louis MAHEU, Les grandes universités de recherche. Institutions autonomes dans un environnement concurrentiel, Montréal, Presses de l'Université de Montréal, 2015. 
d'organisation marchande. Les risques sont grands et tous les pays ne sont pas dans une situation comparable. Les cas les plus préoccupants sont ceux des pays sans tradition universitaire: «il importe [...] de garantir aux systèmes émergents d'enseignement supérieur une qualité, une pertinence et un degré de coopération internationale suffisants, si l'on souhaite qu'ils puissent jouer pleinement leur rôle de pilier dans l'édification de sociétés du savoir ${ }^{55}$ ".

Selon l'UNESCO, l'université du futur reste à inventer, mais l'organisation craint la différentiation des établissements au sein d'un même système. Elle fait aussi état des problèmes engendrés par les demandes contradictoires : promouvoir l'accès le plus large possible au système tout en préservant la qualité des diplômes; établir des normes d'assurance-qualité sans porter atteinte à la liberté universitaire et à l'autonomie des établissements; diversifier les programmes dans un contexte de restrictions budgétaires; et combiner enseignement et recherche. Afin que soit campée la problématique, un rappel historique des idées d'université s'impose.

Le modèle anglais d'université développé par Henry Newman au milieu du XIX ${ }^{e}$ siècle est orienté vers la formation fondamentale. Il déconsidère le savoir immédiatement utile à l'exercice d'une profession. L'université idéale est pour lui «éducation de l'esprit formé par l'étude d'une ou plusieurs disciplines ardues qu'il a partagée avec d'autres étudiants et avec ses maîtres, pour être apte à juger toute variété de savoir nouveau et par là même tendre vers une authentique appréhension du monde entier et de l'homme ${ }^{56}$ ». José Ortega y Gasset ${ }^{57}$ actualise cette thèse : la fonction de l'université est la transmission de la culture. La recherche doit se faire ailleurs, mais elle peut inspirer l'enseignement. Cette idée d'université rappelle le cursus des collèges classiques du Québec d'avant la Révolution tranquille.

Wilhelm Von Humboldt, fondateur de l'Université de Berlin en 1810, propose le modèle allemand, qui implique la combinaison de l'enseignement et de la recherche, leur autonomie par rapport aux contraintes politiques et économiques, la formation par la recherche et la division facultaire. L'autonomie interne touche l'indépendance des sciences par rapport à la philosophie dont l'ambition de systématisation porte atteinte à leur particularisme. L'autonomie externe est la quête désintéressée du savoir et l'élaboration de la science sans considération d'utilité et sans que l'État, l'Église

$$
4+4
$$

55. UNESCO, Vers les sociétés du savoir..., op. cit., p. 89.

56. Owen CHADWICK, Newman, Paris, Cerf, 1982, p. 82.

57. José Ortega y Gasset, Mission of the University, Princeton, Princeton University Press, 1944. 
et la société exigent quoi que ce soit des universités ${ }^{58}$. Karl Jaspers associe pour sa part recherche, formation professionnelle et culture: «One cannot be cut off from the others without destroying the intellectual substance of the university ${ }^{59}$ ". L'université pose ainsi les bases de toute formation puisqu'elle vise le développement d'une pensée scientifique ${ }^{60}$. Les universités contemporaines se réclament de l'héritage humboldtien au chapitre de leur organisation facultaire et de la combinaison de l'enseignement et de la recherche, mais elles s'en éloignent en ce qui a trait à la question de l'autonomie. La notion d'utilité inhérente à l'université actuelle contribue à associer davantage le modèle à la conception étatsunienne.

Celle-ci, hybride des genres précédents mais les transgressant au chapitre de l'utilitarisme, évolue au rythme des conjonctures pour s'adapter aux contraintes et aux occasions du moment et du lieu. Le premier mouvement débute avec le Morill Act de 1862, qui permet la création des universités d'État, préparant à l'exercice des professions. Le soutien accordé à la recherche universitaire par le gouvernement fédéral des États-Unis à compter de la Deuxième Guerre mondiale met en ouvre le second mouvement, consacrant l'utilité de la recherche. La multiversity se développe ensuite, concept évoquant un univers fractionné en de multiples éléments. Ce n'est pas là un idéal aux yeux de Clark Kerr, président de l'Université de Californie de 1958 à 1967, mais un modèle conforme à ce qu'il observe. Son idéal se définit plutôt de manière éclectique :

A university anywhere can aim no higher than to be as British as possible for the sake of the undergraduates, as German as possible for the sake of the graduates and the research personnel, as American as possible for the sake of the public at large - and as confused as possible for the sake of the preservation of the whole uneasy balance ${ }^{61}$.

Autre figure de proue de cette conception, Burton Clark ${ }^{62}$ observe une culture entrepreneuriale qui reflète la volonté d'adaptation des universités aux normes de la performance ${ }^{63}$. Michael Gibbons pose cette hypothèse : la pertinence des activités universitaires devient un attribut dont la preuve doit

$$
\div+\div
$$

58. Alain RENAUT, Les révolutions de l'université, Paris, Calmann-Lévy, 1995.

59. Michael ALLEN, The Goals of Universities, Philadelphia, Society for Research into Higher Education, 1988 , p. 19.

60. Karl JASPERS, The Idea of the University, London, Peter Owen, 1959.

61. Clark KERR, The Uses of the University, Cambridge, Harvard University Press, 1963.

62. Burton ClarK, Creating Entrepreneurial Universities, New York, IAU Press, 1998.

63. Burton CLARK, «L'université entrepreneuriale. Nouvelles bases de la collégialité, de l'autonomie et de la réussite ", Gestion de l'enseignement supérieur, vol. 13, n 2, p. 9-26. 
être apportée de façon continue et les impératifs économiques sont la référence absolue. Il précise : «cette vision de la pertinence de l'université, jugée à l'aune de sa contribution au développement économique, constitue un changement radical par rapport au point de vue et aux valeurs défendues naguère par les disciples de Humboldt et Newman ${ }^{64}$ ".

L'adhésion ou non des universités québécoises à ce modèle est la trame qui alimente le discours critique de plusieurs universitaires. Gilles Labelle ${ }^{65}$ croit que la plupart des ouvrages consacrés à la crise de l'université au Québec répètent les mêmes observations: la distance entre l'université et le marché mondialisé disparaît; l'université s'éloigne de sa vocation humaniste et renonce à former des esprits éclairés pour se conformer à un rôle d'usine à diplômes. Michel Freitag ${ }^{66}$ pose le problème en considérant la dimension institutionnelle de l'université. Avant d'être une organisation, l'université est une institution. L'institution se définit par sa finalité: cela implique une légitimité et une autonomie. L'organisation se définit par les moyens mis en œuvre pour atteindre un but. Or, dans l'université actuelle, le savoir-faire instrumental compte avant tout. L'efficience est devenue la finalité autosuffisante. Gilles Gagné ${ }^{67}$ pousse le raisonnement jusqu'à dégager les axes du programme de réforme de l'université, défini par l'OCDE. On revoit l'organisation de l'institution universitaire selon les modalités d'un nouveau mode de production des connaissances. Gagné ramène à trois traits cette orientation: «l'université doit être ordonnée par sa fonction de recherche plutôt que par sa fonction d'enseignement; la recherche doit viser l'innovation plutôt que la synthèse; l'innovation doit procéder sur le terrain des connaissances appliquées plutôt que sur celui du savoir théorique ${ }^{68}$ ». Ces traits sont les axes d'un programme pour gestionnaires visant à inféoder les universités aux besoins du marché mondialisé.

Yves Gingras ${ }^{69}$ attribue ce discours à une vision mythique d'une mission que l'université se serait donnée au moment de sa création. Réalités institutionnelles et réalités organisationnelles se bousculent plutôt aux portes de l'université qui, à différentes époques, actualise sa mission par la réinven-

$$
4+4
$$

64. Michael GIBBONS, L'enseignement supérieur au XXI siècle, Washington, Banque mondiale, 1998, p. 2. 65. Gilles LABELLE, "Compte rendu du livre de Pierre Hébert. La nouvelle université guerrière ", Recherches sociographiques, vol. 43, no 2, 2002, p. 427-429.

66. Michel Freitag, Le naufrage de l'université, Québec; Paris, Nuit blanche éditeur; La Découverte, 1995.

67. Gilles GAGNÉ, "La restructuration de l'université. Son programme et ses accessoires», Société, $\mathrm{n}^{\text {os }} 24-25$, p. 31-53.

68. Ibid., p. 39.

69. Yves GINGRAS, «L'université en mouvement », Égalité, nº 50, 2004, p. 13-28. 
tion de différents compromis à la faveur d'une durée. Il rappelle que universitas signifie "corporation de maîtres et d'élèves " et que les premières universités forment des praticiens, juristes et médecins. Les universités entretiennent d'étroites relations avec l'Église et l'État. L'université répond aux demandes externes par différentes adaptations:

La tension entre le désir d'autonomie de l'université et les tentatives de contrôle par l'État (et auparavant par l'Église) a toujours existé et constitue une caractéristique fondamentale et inscrite dans la longue durée de l'histoire des universités dont la vie propre (interne) n'est pas séparable de la vie sociale (externe $)^{70}$.

À l'échelle de l'histoire millénaire des universités, la mission de transfert scientifique, inhérente au phénomène d'instrumentalisation du savoir, est cependant récente. Son émergence consacre une rupture. L'institution universitaire, autonome et stable en ce qu'elle préexiste à ses membres et qu'elle subsiste après eux ${ }^{71}$, demeure soumise à des normes explicites, les instructions, et à des normes implicites, les traditions, qui limitent la contrainte tout en la légitimant. Deux de ces normes résistent à l'épreuve du temps. D'abord, l'université a toujours été liée à l'idée d'association. À l'origine, des étudiants se regroupent. Ensuite, maîtres et élèves font de même, parfois ensemble, parfois en parallèle. Ils se donnent des règles d'entraide qui garantissent leur protection face aux menaces extérieures (le savoir présente un aspect hérétique) et réglementent l'exercice autonome de l'activité qui est leur raison d'être: l'étude ${ }^{72}$. Les fonctions de l'étude varient et se confondent : savoir utile en lui-même, utile à une pratique spécialisée ou consacrant une position sociale. Ces deux normes ont cependant toujours coexisté : l'idée d'association renvoie à une logique organisationnelle (le comment), la finalité éducative renvoie à une logique institutionnelle (le pourquoi).

Une rupture apparaît au XIX ${ }^{e}$ siècle avec une actualisation de la mission de l'université : c'est la première révolution universitaire. Jusque-là, le savoir se présente comme un corpus fermé, une masse de vérités révélées et marquées souvent du sceau religieux. Il n'est pas possible de remettre en question ce savoir, il faut l'intégrer. La recherche n'est pas une réalité signifiante: "Outre que les notions mêmes de recherche et de découvertes scientifiques n'étaient guère familières aux hommes de ce temps, l'idée que

$$
\div+\div
$$

70. Ibid., p. 14 .

71. Olivier Reboul, La philosophie de l'éducation, Paris, Presses universitaires de France, 1992.

72. Christophe CHARLE et Jacques VERGER, Histoire des universités, Paris, Presses universitaires de France, 1994. 
les universités auraient pu promouvoir l'association féconde de l'enseignement et de la recherche [...] leur était [...] carrément étrangère ${ }^{73}{ }$. C'est vrai depuis les origines jusqu'au XIX ${ }^{\mathrm{e}}$ siècle. Les sciences se développent alors hors de l'université, par l'action d'individus commandités par des mécènes ou eux-mêmes indépendants de fortune ${ }^{74}$. L'activité de recherche intègre graduellement l'université sous l'impulsion du modèle humboldtien. Aux ÉtatsUnis, les universités Harvard et Columbia font de la recherche une dimension essentielle de leur mission. Vers la fin du XIX siècle, les universités de Chicago et Johns Hopkins "donnent à cette perspective un élan décisif ${ }^{75}$ ".

Une seconde révolution universitaire survient au $\mathrm{XX}^{\mathrm{e}}$ siècle, aux États-Unis d'abord. Le rapport Science: The Endless Frontier ${ }^{76}$ consacre le modèle. Gilbert Hottois ${ }^{77}$ l'associe à une conception linéaire des relations entre science et société, une conception marquée par ces caractéristiques: la recherche fondamentale, libre et financée par l'État, doit se faire à l'université; elle permet la réalisation de produits utiles au développement d'entreprises. Certes, les universités ont toujours entretenu d'étroites relations avec l'Église d'abord et avec l'État ensuite. Cependant, ce moment aux ÉtatsUnis, qui est celui de l'émergence des premières politiques scientifiques nationales, confirme la mission de transfert scientifique qui se résume à cette idée : la transmission à des usagers de savoirs et de savoir-faire scientifiques utiles. La mission de transfert scientifique de l'université se précise au cours de la seconde moitié du XXe siècle avec le modèle de la multiversity, archétype de l'université de classe mondiale.

Au Québec aussi, l'université du futur reste à inventer et la différenciation des établissements est un enjeu d'actualité. Le Québec demeure un des rares endroits où il n'y a pas d'universités privées, malgré que des demandes en ce sens soient parfois adressées au ministère de l'Éducation, comme nous le confie un détenteur d'enjeux. La volonté de différenciation existe cependant; en témoigne l'implosion de la Conférence des recteurs et des principaux des universités du Québec (CREPUQ) à compter de 2013, après cinquante ans d'existence, alors qu'il n'est plus possible pour les universités de tenir un discours unique sur les modalités (la gestion et la gouver-

$$
\div+\div
$$

73. Jacques VERGER, "Les universités à l'époque moderne", Gaston MiALARET et Jean VIAL (dir.), Histoire mondiale de l'éducation, vol. 2, Paris, Presses universitaires de France, 1981, p. 266.

74. Daniel Boorstin, Les Découvreurs, Paris, Robert Laffont, 1986.

75. Manuel CRESPO, "Une nouvelle révolution universitaire? L'échange des rôles de la triade universitéentreprise-État", Revue des sciences de l'éducation, vol. 24, n² 2, 2003, p. 380.

76. Vannevar BusH, Science. The Endless Frontier, Report to the President, Washington D.C, 1945.

77. Gilbert HОтTOIS, La science. Entre valeurs modernes et postmodernité, Paris, Vrin, 2005. 
nance universitaires) et les principes modelant l'institution/organisation (les missions de l'université $)^{78}$. Le printemps érable de 2012 , outre l'enjeu des droits de scolarité, met en lumière une critique du modèle de développement privilégié : détournement de l'université vers des fins mercantiles ${ }^{79}$ et logiques d'individualisation, d'enrichissement personnel et de développement économique qui animent les universités ${ }^{80}$. La solution de remplacement est inscrite dans le référentiel porté par l'UNESCO. Paradoxe cependant, la ministre de l'Éducation en poste lors du printemps érable est maintenant représentante du Québec au sein de la Délégation permanente du Canada à l'UNESCO, une organisation qui milite pour la gratuité scolaire ${ }^{81}$.

En 2015, le référentiel dominant dans les universités du Québec demeure celui de l'économie du savoir. L'ouvrage de Robert Lacroix et Louis Maheu, auquel nous avons fait référence plus tôt, se présente comme un argumentaire de ce modèle. Intitulé Les grandes universités de recherche. Institutions autonomes dans un environnement concurrentiel, le livre insiste sur le fait que "si toutes les universités vouent un grand respect aux connaissances et à l'esprit de recherche, elles ne sont pas pour autant identiques quant aux missions et aux fonctions premières qu'elles assument ${ }^{82}$ ". Sans déprécier nommément les "petites universités ", les auteurs militent pour un réinvestissement dans les grandes universités de recherche susceptibles de bien se classer dans les palmarès mondiaux. Il y a, dans ce discours, une volonté de concentrer les ressources « là où ça rapporte ». Les auteurs empruntent cependant un raccourci intellectuel lorsqu'ils écrivent que le gel des droits de scolarité crée un sous-financement chronique du système: "Il s'agit là d'une grave erreur qui a déjà des impacts notoires sur la qualité des universités québécoises et qui risque d'hypothéquer lourdement le futur du Québec ${ }^{83}$ ", prétendent-ils.

À cela s'ajoute une différenciation selon les facultés et les domaines d'enseignement et de recherche. L'École de technologie supérieure par exemple, dont le slogan est "Le génie pour l'industrie », peut prétendre à

$$
\div \div
$$

78. Reginald HARVEY, "Après la CREPUQ. Il n'est plus possible de tenir un discours unique », Le Devoir, 17 août 2013, http://www.ledevoir.com/societe/education/385120/il-n-est-plus-possible-de-tenir-undiscours-unique (7 mars 2015).

79. Éric MARTIN et Maxime Ouellet, Université inc., Montréal, Lux, 2011.

80. Pierre-Luc BRISSON, Après le printemps, Montréal, Poètes de brousse, 2012.

81. Michel CORBEIL, "Line Beauchamp nommée à l'UNESCO à Paris ", Le Soleil, 10 septembre 2014, http://www.lapresse.ca/le-soleil/actualites/politique/201409/10/01-4799004-line-beauchamp-nommeea-lunesco-a-paris.php (7 mars 2015).

82. Robert LACROIX et Louis MAHEU, Les grandes universités de recherche..., op. cit., p. 10.

83. Ibid., p. 301. 
cette étiquette sans émouvoir les critiques du modèle, alors qu'une université dont le slogan serait «Le savoir au service du marché mondialisé» risquerait de s'attirer les foudres. D'ailleurs, c'est une opposition populaire qui a fait reculer une grande université de recherche dans son projet d'implanter sur son campus un supermarché-école, en $2006^{84}$.

\section{CONCLUSION}

Le rapport de l'UNESCO sur les sociétés du savoir de 2005 est le premier rapport mondial que l'organisation publie. Les rapports précédents sont des documents sectoriels. C'est un texte normatif qui présente une vision $d u$ monde alternative, une solution de remplacement au référentiel de l'économie du savoir promu par l'OCDE et souvent présenté comme la seule option possible, la voie pragmatique adaptée à la réalité qui caractérise notre monde. Le référentiel de l'UNESCO est une utopie, au sens de la définition du Larousse: "construction imaginaire et rigoureuse d'une société, qui constitue, par rapport à celui qui la réalise, un idéal ou un contre-idéal ${ }^{85} \%$. On peut se demander, en conséquence, si le modèle rival, celui de l'économie du savoir, n'est pas une dystopie, car il repose sur des valeurs et des normes marquées par l'individualisme: le savoir, considéré comme une ressource stratégique au service des intérêts nationaux et de la croissance économique, est privatisé. Rappelons-le cependant: ces référentiels sont des idéaux-types que l'on ne retrouve pas intégralement dans l'univers social; ce sont des vues de l'esprit qui permettent de mieux comprendre les phénomènes complexes.

En ce qui a trait à la gouvernance de la science, l'UNESCO propose le modèle du public avec décisions partagées que suggère l'image du triangle (lieux de la science, État et entreprises, société civile) avec à l'interface un espace public de débat, de concertation ou de confrontation. Au Québec, on observe que les gouvernements formés par le Parti québécois sont plus enclins que ceux formés par le Parti libéral à mettre en place des instruments de consultation et de délibération sur les grands enjeux de leurs politiques scientifiques. La science est une responsabilité partagée entre les gouvernements fédéral et provinciaux. L'action du gouvernement conservateur en science et en technologie va à l'encontre des principes de gouvernance. Il est possible de l'associer à un mode de gouvernement hiérarchique, contraignant et dogmatique. Le dogme est caractérisé par son «idéologie de

$$
4 \div 4
$$

84. CADEUL, Revue de presse Sobey's, 16 février au 9 mars 2006, http://www.cadeul.ulaval.ca/cadeul/doc/ Sobeys_revue\%20sobeys\%20du\%2016-02-06\%20au\%2009-03-06.pdf (7 mars 2015).

85. LE LAROUSSE, «Utopie », http://www.larousse.fr/dictionnaires/francais/utopie/80825 (20 avril 2014). 
droite crypto-évangéliste». On observe néanmoins dans la société civile un tournant délibératif en ce qui concerne les rapports entre science et société.

Sur le plan de l'organisation de la science, le rapport de l'UNESCO rend compte d'une finalité, actualisée au rythme des mouvements qui secouent le monde, qui s'exprime par ces deux dimensions : paix et développement (le pourquoi de la science). Partage et collaboration (le comment de la science) sont les vecteurs qui permettent de fonder les sociétés du savoir. Dans le contexte du référentiel de l'économie du savoir, les normes de communalisme et de désintéressement sont niées, elles qui constituent l'ethos de la communauté scientifique, bien qu'elles soient fréquemment transgressées. Au Québec, la recherche scientifique est de plus en plus orientée, mais elle l'est parfois dans un sens qui sert le bien commun. Quelques projets institutionnels sont mis en œuvre, mais les initiatives relèvent davantage de l'engagement individuel de certains chercheurs.

L'évolution de l'enseignement supérieur témoigne que deux normes ont toujours coexisté : l'idée d'association renvoie à une logique organisationnelle (le comment), tandis que la finalité éducative renvoie à une logique institutionnelle (le pourquoi). Il n'existe pas d'âge d'or des universités. Il n'y a pas non plus un contexte qu'il nous faut reproduire ici et maintenant. D'ailleurs, l'université d'antan, celle du milieu du XXe siècle au Québec, est dogmatique, sexiste et accessible aux seules personnes qui en ont les moyens financiers. Au moment où personne, ou presque, n'adhère plus au modèle de l'université comme tour d'ivoire, l'instrumentalisation du savoir et de l'université est un phénomène avec lequel il nous faut composer. Mais une science et une université instrumentalisées pour quoi et par qui ? Dans nos sociétés mondialisées incertaines et instables, la participation active de chacun au nouvel espace public mondial est une condition de paix et de développement. Cela est possible grâce au partage et à la collaboration. 